\title{
Typologies des villages et pratiques d'élevage dans le Badakhshan (nord-est de l'Afghanistan)
}

\author{
M. Bouy ${ }^{1^{*}}$, J. Dasnière ${ }^{1^{*}}$
}

BOUY (M.), DASNIÈRE (J.). Typologies des villages et pratiques d'élevage dans le Badakhshan (nord-est de l'Afghanistan). Revue Elev. Méd. vét. Pays trop., 1994, 47 (2) : 245-256

Une étude du système d'élevage a été menée dans deux vallées du Badakhshan, région du nord-est de l'Afghanistan. La première partie de cette élude a consisté à établir une typologie des villages tenant compte de leurs aspects agro-écologiques et humains. Cette typologie a mis en évidence trois groupes principaux : fond de vallée irriguée, montagne irriguée et montagne sèche. En se basant sur cette typologie, une étude des pratiques d'élevage touchant à l'alimentation et à la reproduction des bovins et des petits ruminants est présentée. Ces pratiques montrent la forte association de l'élevage bovin à l'agriculture, et l'exploitation exclusive du domaine montagnard par les petits ruminants. Le rôle des pratiques collectives est abordé dans chaque cas.

Mots clés : Ane - Bovin - Caprin - Cheval - Poule - Ovin - Région et altitude - Vallée - Village - Association agriculture-élevage - Méthode d'élevage - Reproduction - Alimentation - Fourrage - Engraissement - Nomadisme - Pastoralisme - Economie de l'élevage - Afghanistan.

\section{INTRODUCTION}

Entre juillet 1989 et juin 1990, l'ONG "Vétérinaires sans frontières" a mis en œuvre un projet de soutien de l'élevage dans le Badakhshan, province du nord-est de l'Afghanistan. La première partie de cet article est consacrée à une description de la zone d'étude et des principales caractéristiques de l'élevage. La diversité des situations a conduit à construire une typologie des villages sur laquelle est basée, dans la deuxième partie de l'article, la présentation des différentes pratiques d'élevage. L'Afghanistan est un pays ruiné par la guerre. Aucune information fiable n'est disponible concernant la démographie et les activités agricoles. Cette situation particulière explique le caractère un peu frustre de certaines données présentées dans cet article.

\section{PRÉSENTATION DE LA ZONE D'ÉTUDE}

\section{Le Badakhshan et la région de Jurm (carte 1)}

Le Badakhshan ( $36^{\circ} 5$ de latitude nord) connaît un climat semi-aride à hiver froid (4) et reçoit des pluies printanières plus tardives que d'autres régions d'Afghanistan,

1. Vétérinaires sans frontières, ERAC, 14 avenue Berthelot, 69361 Lyon Cedex 07, France.

*Adresse actuelle : Léo de Bosdarros, 64800 Haut-de-Bosdarros, France.

Reçu le 5.9.1991, accepté le 14.6.1994.

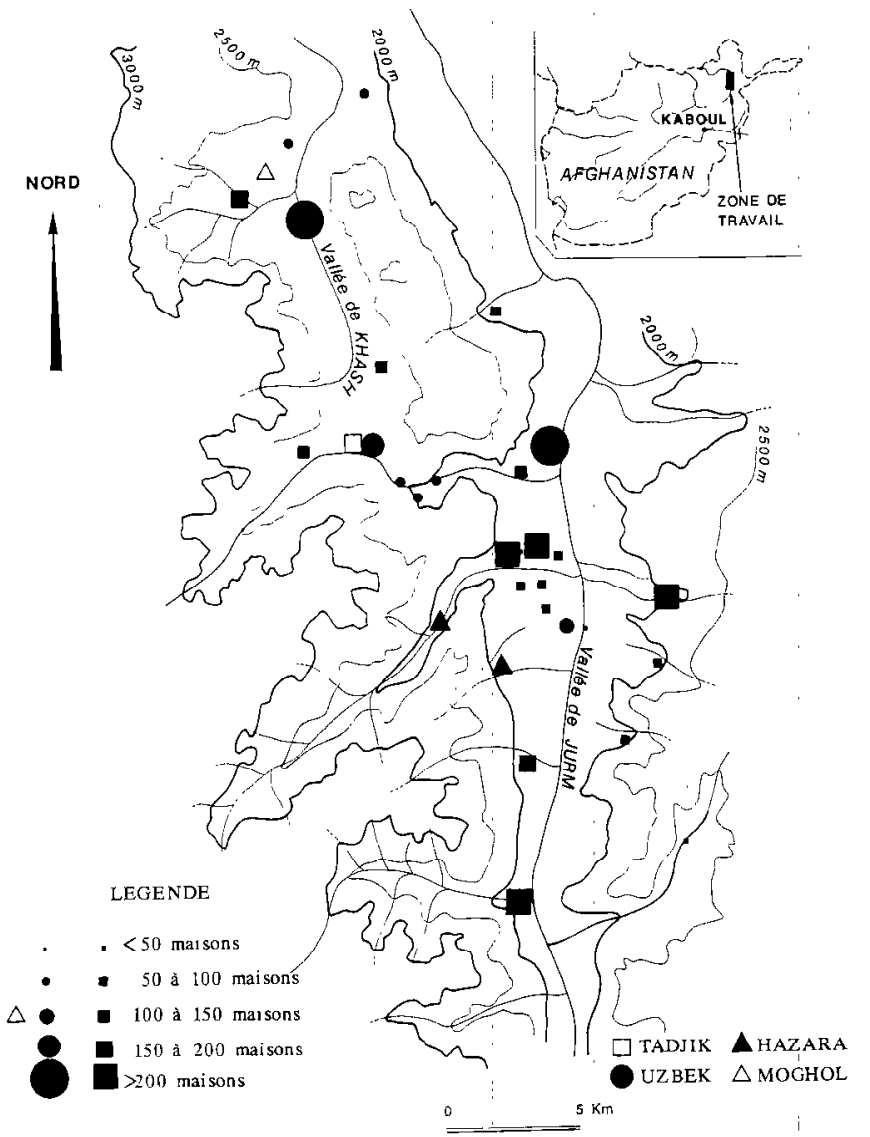

Carte 1 : Vallées de Jurm et de Khâsh : taille et composition ethnique des uillages.

permettant un développement important des cultures pluviales appelées lalmi (2). Cette province est un "véritable muséum ethnolinguistique" (16) car la région a servi de refuge pour le groupe de l'ancien peuplement (Tadjik) qui vit maintenant aux côtés de ceux arrivés plus récemment (Uzbeks, Hazâras, Moghols, Pashtouns). La zone de travail a concerné 30 villages situés dans les vallées de Jurm et de Khâsh. Ces villages comptent chacun en moyenne 140 maisons (extrêmes 10-350). En prenant une moyenne de 7 personnes par famille (17), l'ensemble des villages étudiés regroupe environ 27000 personnes. Tout au long de cet article, le terme de "famille" et "maison" sont utilisés pour décrire l'unité sociale la plus petite, basée sur le groupe domestique. La vallée de Jurm, longue d'une centaine de kilomètres, est orientée sur un axe nord-sud. Elle s'enfonce de plus en plus étroite vers le 
sud, entre les premiers contreforts de l'Hindu-Kush. Dans sa partie la plus large, trois kilomètres séparent les deux flancs montagneux. La rivière qui y coule a creusé un lit profond, et devient de ce fait inutilisable pour l'irrigation. Les 19 villages étudiés occupent les vingt premiers kilomètres et sont situés entre 1500 et $2300 \mathrm{~m}$ d'altitude. Ils vivent essentiellement de la production de blé cultivé sur des sols de loess. Au-dessus de $3000 \mathrm{~m}$, les estives, appelées aylâq, sont exploitées par les petits ruminants. La vallée de Jurm est principalement peuplée de Tadjiks, mais l'on y trouve trois villages uzbeks et deux villages hazâras. Deux cluses reliées par un plateau d'altitude définissent la vallée de Khâsh. Les onze villages de cette vallée se situent entre 1850 et $2300 \mathrm{~m}$, et sont surtout peuplés d'Uzbeks. Toutefois, il y a trois villages tadjiks et un village moghol.

\section{L'espace-ressource des villages}

\section{Les surfaces de fond de vallée}

Elles regroupent les surfaces situées à proximité des villages et comprennent le domaine irrigué et le domaine pluvial.

Les zones irriguées naturellement ou par l'homme regroupent le dasht irrigué, les marzars et les jardins.

Le dasht irrigué, en propriété privée, est l'ensemble des champs irrigués labourés : il produit du blé, de l'orge, du trèfle persan (Trifolium resuspinatum), de la luzerne et des melons d'eau.

Les marzars sont, soit privés, soit collectifs, et sont des zones naturellement irriguées, impropres à la culture. Elles sont toujours pâturées et parfois fanées. Ces surfaces se situent en bordure des torrents ou dans des zones de résurgence. Situé généralement dans l'enceinte de la maison, le jardin associe potager et verger (abricotiers, pêchers, pommiers, mûriers).

Le domaine pluvial de fond de vallée comprend les parcours et le dasht lalmi. Ce dernier est d'une grande importance malgré des rendements aléatoires. II est toujours en propriété privée. Labouré, il permet la production de blé, d'orge et parfois de pavot. Ces surfaces ne sont pas irrigables, soit du fait de leur situation, soit à cause de l'absence ou de l'abandon des canaux d'irrigation. Les parcours appartiennent généralement au village entier, l'usage en est plutôt collectif, mais les habitations et les bâtiments d'élevage qui s'y trouvent sont privés.

\section{Le domaine montagnard}

Le domaine montagnard comprend aussi bien des espaces cultivés que des surfaces de parcours. Les surfaces labourées correspondent à des cultures pluviales (lalmi) de blé, d'orge, d'avoine et parfois de pavot. Ces surfaces restent en propriété privée. En ce qui concerne

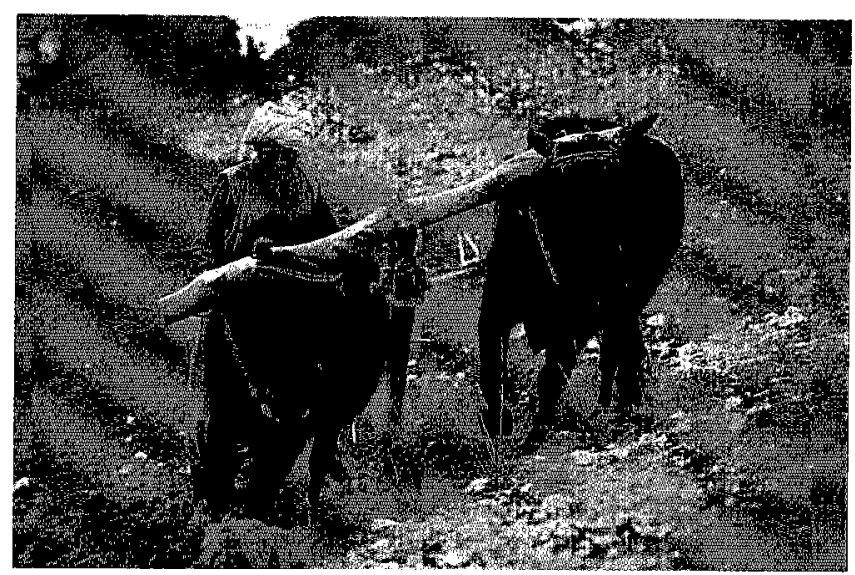

Photo 1 : Afghanistan. Iahour des champs non irrigués (mars 1990).

les parcours, il est possible de distinguer la montagne proche et les zones d'estives (aylâq). Ces surfaces sont utilisées pour le pâturage du gros bétail et aussi pour la constitution de réserves fourragères. La montagne proche est utilisée de décembre à mai tant que la couverture neigeuse n'empêche pas le pâturage ; c'est la période où les troupeaux rentrent tous les soirs au village. Comme pour les parcours de fond de vallée, les pâturages d'altitude appartiennent à la communauté villageoise mais les bâtiments qui s'y trouvent, véritables villages d'altitude dans certains cas, sont en propriété privée.

\section{Principales caractéristiques de l'élevage}

\section{Les bovins}

La très granide majorité des animaux est de race locale. Le terme qui désigne ce cheptel est rasak, par opposition aux bovins lewandi désignant les animaux de race de grande taille importés d'autres régions d'Afghanistan ou de l'étranger. La guerre a interrompu ces importations, et ces animaux lewandi semblent absents de la région à l'heure actuelle. On trouve quelques bovins du Nouristan (province au sud du Badakhshan), animaux reconnaissables par leurs grandes cornes en lyre, et dénommés jadidi. La race locale est un zébu à la bosse peu proéminente. Les animaux sont de petite taille (respectivement $1 \mathrm{~m}$ et $1,15 \mathrm{~m}$ au garrot pour les vaches et les boeufs). IIs ont un profil rectiligne et la robe est le plus souvent noire ou fauve très foncée ; les robes pie-noir et froment clair se rencontrent aussi. Les cornes sont courtes. Par barymétrie, il est possible d'estimer les poids moyens des bovins à $190 \mathrm{~kg}$ pour une vache de 4 ans, et à $300 \mathrm{~kg}$ pour un boeuf de plus de 5 ans. Les productions essentielles sont le travail et le lait. L'utilisation des bovins à des fins bouchères est liée à la réforme des animaux (vieillesse, maladie ou accident). Dans la langue farsi uti- 
lisée par les Tadjiks, le mot gao désigne les bovins en général. Mais dans le langage courant, ce terme est utilisé pour nommer les bœufs, alors que le mot barzago (= bœuf) est très peu employé. Cette substitution montre que le boeuf, animal de trait, est au centre des préoccupations concernant l'élevage bovin.

Douve du foie et charbon symptomatique sont les deux pathologies essentielles des bovins. La peste bovine et la fièvre aphteuse, connues des paysans, ne sont pas considérées comme un problème dans la région. La toxocarose aggrave systématiquement la sous-nutrition dont pâtit le veau dans les premiers mois de sa vie (concurrence entre la traite et la tétée).

\section{Les ovins et les caprins}

Dans la région de Jurm, on rencontre essentiellement deux races ovines: Turki et Gadi. "Le Turki est une des deux races à croupe grasse de l'Afghanistan. Les moutons Turki sont parmi les plus grands moutons du monde (hauteur au garrot : $76 \mathrm{~cm}$ pour les brebis, $86 \mathrm{~cm}$ pour les béliers). C'est par excellence la race à viande de l'Afghanistan. La toison des moutons Turki est très semblable à celle des moutons sauvages. Les fibres sont courtes et la toison est principalement composée de jarres, sur un mince fond de laine. La toison a donc peu de valeur commerciale" (13).

Dans la pratique, toutes les productions potentielles sont valorisées : les animaux de 15 mois sont vendus pour la viande à des intermédiaires qui les emmènent à pied vers les grandes villes (Kaboul, Kunduz). Un sevrage précoce rend possible la traite, et les produits laitiers jouent un rôle important dans l'alimentation, tant estivale (crème, yaourt) qu'hivernale (beurre, kurut (3)). L'artisanat domestique repose principalement sur le travail de la laine. Celle de la tonte de printemps est filée, puis tissée pour la fabrication de nappes, de sacoches, de sacs à céréales et de sangles. La laine de la tonte d'automne, plus courte et plus épaisse, sert à confectionner les tapis de feutre (namad) présents dans chaque maison.

La race Gadi est de plus petit format $(65 \mathrm{~cm}$ au garrot, 25 $\mathrm{kg}$ pour une brebis adulte). C'est une race à queue grasse, et non à croupe grasse comme le Turki. Au Badakhshan, le lieu d'élection de cette race se situe à Zebâk à $200 \mathrm{~km}$ au sud-est de Jurm. Cette race a la réputation de faire deux agnelages par an, elle est élevée aux mêmes fins que le Turki. Cependant, les animaux destinés à la boucherie sont commercialisés localement et non à distance comme dans le cas précédent. II est intéressant de noter la persistance de l'utilisation des mots tokhli, shishak et châri pour désigner respectivement les animaux de moins d'un an, de 1 à 2 ans, et de 2 à 3 ans. Ces trois termes désignent en effet aussi les trois tailles de socs d'araire (8).

Dans la région de Jurm, une troisième race est présente de façon anecdotique : il s'agit du mouton Arabi (Afghanistan), grande race à croupe grasse et à laine de très bonne qualité. Un troupeau Arabi hiverne à proximité de
Jurm durant la mauvaise saison. II n'y a qu'une seule race de chèvre, de petit format $(25-30 \mathrm{~kg}$ pour une chèvre adulte), et qui possède un pelage fourni, généralement de couleur noire. Les chèvres sont élevées pour leur aptitude mixte : lait, viande et poil. Les parasitoses internes (strongyloses digestives et respiratoires, douve) et externes (tiques, gale) atteignent de façon chronique les petits ruminants. La fièvre charbonneuse sévit sous forme d'épidémie durant les mois d'été, et est responsable de taux de mortalité avoisinant les 25 p. 100 . Les chèvres sont beaucoup plus sensibles aux bronchopneumonies infectieuses qui apparaissent en hiver, largement favorisées par le parasitisme et le confinement dans les bâtiments.

\section{Ânes et chevaux}

Les ânes jouent un rôle primordial pour le bât et assurent l'essentiel du transport des denrées, des récoltes et du fourrage. En période de guerre où la circulation des camions est sporadique, cette fonction de transport est vitale. On rencontre deux types d'ânes : le premier concerne des animaux de petite taille (environ $1 \mathrm{~m}$ au garrot) au poil gris ou noir, avec parfois les pattes zébrées. Le second type correspond à des ânes plus grands (1,30 m au garrot), dont la robe est la plupart du temps blanche. Plaies de harnachement et pathologie respiratoire représentent les pathologies principales.

Le Badakhshan n'est pas une région d'élevage de chevaux qui sont importés en majorité de provinces plus à l'ouest (Kunduz, Mazar-E Sharif). Aussi est-il difficile de reconnaître une ou plusieurs races particulières. Les vallées au sud de Jurm (Khâshtak, Jokham) possèdent plus de chevaux et l'on y rencontre des animaux de très petite taille ( $1 \mathrm{~m}$ à $1,20 \mathrm{~m}$ au garrot). Si les chevaux sont parfois utilisés pour le bât, ils restent avant tout un signe de richesse, et sont élevés par les notables, grands propriétaires, pour la monte lors des déplacements et surtout pour le bozkachi (sport traditionnel en Afghanistan). II semble que le tétanos soit la maladie la plus répandue dans cette espèce.

\section{Les poules}

La race locale est de petit format et porte fréquemment une huppe. Dans un village, plusieurs familles possèdent des poules descendantes d'animaux de race française, importés avant la guerre, et appréciées par la taille plus importante des oeufs. L'effectif de volailles connaît des fluctuations saisonnières : à l'automne, de nombreuses poules sont vendues ou tuées, puis au printemps les bandes sont reconstituées. Cette pratique permet d'éviter de nourrir des animaux l'hiver, et de limiter les conséquences de l'épidémie de maladie de Newcastle qui sévit annuellement à cette période. Environ deux familles sur trois possèdent des poules et chaque maison en a entre 
2 et 6 . L'idée que les poules sont élevées en se nourrissant de rien est fausse puisque durant tout l'hiver, les femmes leur distribuent tous les matins du blé ou du pain sec. Les volailles sont donc véritablement élevées et non livrées à elles-mêmes.

\section{Typologie des villages}

\section{Objectif et méthode}

Les vallées de Jurm et de Khâsh présentent une grande diversité tant naturelle qu'humaine : altitude allant de 1700 à $3500 \mathrm{~m}$, opposition domaine irrigué/pluvial, labouré/pâturé, patchwork ethnique, etc. La compréhension des pratiques d'élevage implique de tenir compte de cette diversité sans se perdre pour autant dans la situation particulière de chaque village. Il est nécessaire d'ajouter qu'au sein des villages existent de grandes différences d'une famille à l'autre. Ce point sera abordé ultérieurement.

La typologie des villages a été élaborée en tenant compte de l'espace-ressource de chaque village (tableau I), situation géographique (appartenance à telle ou telle vallée, altitude, localisation), origine ethnique des habitants et taille de la population. Les variables concernant l'espace-ressource ont été codées de façon semi-quantitative : absence, surface peu importante, surface importante. Par rapport à cette méthode, trois remarques s'imposent:

- ce codage a été réalisé en recoupant nos observations avec des enquêtes orales informelles auprès des paysans. Dans ces conditions, il est impossible de préciser plus finement l'étendue de chaque type de surface ;

- les valeurs attribuées à chaque ressource correspondent à une moyenne et ne reflètent pas l'inégalité foncière qui existe au sein de chaque village ;

- la méthode de codage donne le même poids à des surfaces d'étendues très différentes ; ainsi, les marzars correspondent souvent à moins d'un dixième des zones irriguées. Ces pâturages jouent un rôle important dans l'alimentation du bétail et il semble légitime que leur importance soit prise en compte dans le cadre de l'étude d'un système d'élevage.

Un tableau comprenant 30 individus (villages) et 12 variables a ainsi été construit (tableau I). Les variables sont soit semi-quantitatives (espace-ressource, altitude, population), soit qualitatives (vallée, situation, ethnie). L'analyse de correspondance multiple (ACM) a semblé être la méthode la plus appropriée pour la compréhension d'un tel tableau.

\section{Analyse des résultats}

L'axe 1 est défini par les variables altitude, montagne proche et aylâq. II oppose les villages de fond de vallée utilisant peu la montagne et ne possédant pas d'estives aux villages de montagne. Trois variables contribuent à la définition de l'axe 2 (dasht irrigué, marzars privés et col- lectifs) qui oppose les villages possédant de nombreuses surfaces irriguées à ceux fonctionnant essentiellement en système pluvial. Le plan factoriel 1-2 correspond à un pourcentage d'inertie de $61 \mathrm{p} .100$. On peut considérer que la projection du nuage de points sur le plan 1-2 entraîne une perte d'informations modérée. En effet, dans le cas des $A C M$, et à la différence des AFC, les pourcentages d'inertie de chaque axe sont souvent faibles du fait de la transformation de chaque modalité en variable. La classification des villages en chaque groupe a été effectuée de la façon suivante : pour les villages bien représentés (corrélations élevées sur les axes 1 et 2), c'est la proximité de ces villages sur le plan 1-2 qui a permis de constituer les groupes. Les villages mal représentés ont été rassemblés par défaut dans le $4 \mathrm{e}$ groupe. Les résultats de la projection des 30 villages sur le plan 1-2 de l'ACM permet de définir 4 groupes de villages:

- le groupe 1 rassemble 7 villages de l'agglomération de Jurm et sont de type fond de vallée irriguée ;

- le groupe 2 réunit 7 villages de montagne à tendance "irrigués" et appartiennent tous à la vallée de Khâsh. Ils se situent aux altitudes les plus hautes;

- le groupe 3 comprend 6 villages localisés sur le piémont de la vallée de Jurm et un village de la vallée de Khâsh. Aux altitudes intermédiaires, ces villages disposent de très peu de surfaces irriguées ;

- le groupe 4 est formé par défaut par 8 villages mal représentés sur le plan 1-2. La signification concrète de ces villages est détaillée ci-après.

\section{Interprétation}

\section{Signification des 4 groupes}

Le groupe 1 (fond de vallée plus ou moins irrigué) est entièrement tadjik. Cette ethnie persanophone forme le plus ancien peuplement du Badakhshan. Elle est constituée de paysans sédentaires pratiquant la culture irriguée et l'arboriculture (16). Les habitants des villages du groupe 1 se trouvent donc dans leur zone agro-écologique de prédilection.

Le groupe 2 (montagne irriguée) est essentiellement uzbek, ethnie turcophone qui s'est établie au Badakhshan à partir du XVle siècle (16). Les Uzbeks sont traditionnellement des éleveurs transhumants ou seminomades. Cela explique l'altitude élevée de leurs villages, permettant une exploitation importante du domaine montagnard par l'élevage des petits ruminants. Là encore, les paysans de ces villages habitent une zone agro-écologique qui leur est habituelle. En outre, ils se trouvent dans une situation relativement privilégiée, car l'existence d'une zone irrigable importante leur permet d'associer culture irriguée du blé et élevage transhumant apportant une bonne sécurité face aux aléas climatiques. Cette position favorable est peut-être à mettre en rapport avec la domination politique uzbek qui a existé durant les XVIIle et XIXe siècles (16). 
TABLEAU I Caractéristique des villages.

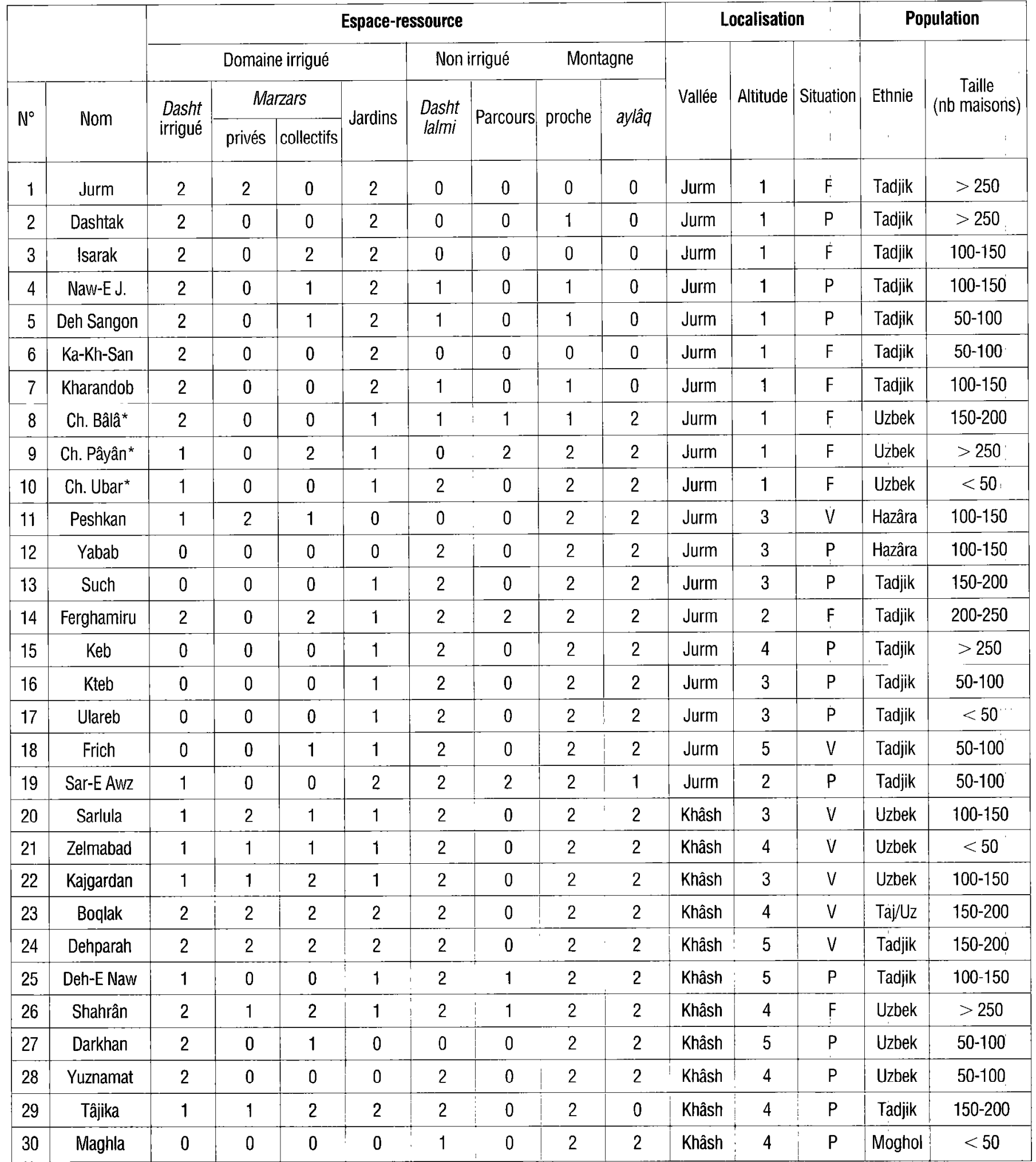


Dans le groupe 3 (montagne sèche), situé dans la zone la plus défavorable, on retrouve 5 villages tadjiks et les 3 villages peuplés par les ethnies minoritaires Hazâras et Moghols. Dans l'ensemble de l'Afghanistan, les Hazâras constituent le groupe de référence négatif aux yeux des autres ethnies; il se situe au bas de l'échelle hiérarchique (6). On retrouve, dans ce cas précis, une cohérence entre le statut social du groupe ethnique et la qualité de son espace-ressource. Cette situation est particulièrement marquée pour le village de Yabab $\left(n^{\circ} 12\right)$ qui ne possède aucune surface irriguée.

Dans le groupe 4 (villages mal représentés sur le plan 12), il est possible de distinguer 2 villages (Sar-E Awz et Ferghamiru) caractérisés par des surfaces en parcours très importantes. Ils se situent aux extrémités nord et sud de la zone d'étude, là où la vallée change de morphologie : ouverture dans un cas, rétrécissement dans l'autre. Ces dcux villages sont géographiquement marginaux par rapport au reste de la zone.

Trois autres villages du groupe 4 se nomment respectivement Chingah bâlâ, Chingah pâyân et Chingah ubar, signifiant Chingah d'en haut, d'en bas et de l'autre rive. Ils ont la particularité d'être situés en fond de vallée à faible altitude, de posséder diverses surfaces irriguées, tout en exploitant de façon importante des estives et des parcours situés loin du village. Cette dernière pratique est à mettre en rapport avec l'origine Uzbek des habitants des Chingah. Or, ces trois villages sont dans la vallée de Jurm, vallée à dominante Tadjik. Ainsi les Chingah ont un espace-ressource géographiquement éclaté, et sont des villages socialement marginaux. Cette marginalité se traduit dans la vie quotidienne des Jurmi par le mépris que les Uzbeks des Chingah subissent de la part des autres habitants de la vallée, alors que ce mépris ne s'exerce guère envers les Uzbeks de la vallée de Khâsh.

Les 3 autres villages appartiennent à la vallée de Khâsh et se trouvent dans des situations intermédiaires entre le domaine irrigué et le domaine pluvial. Aucune explication particulière ne peut être donnée pour comprendre cette situation ; néanmoins il faut noter qu'un de ces villages (Deh-E Naw) était Uzbek autrefois (enquête réalisée en 1992) (12), alors qu'il est Tadjik aujourd'hui.

\section{Configuration des deux vallées}

La vallée de Khâsh présente un ensemble relativement homogène avec 6 villages sur 10 dans le groupe 2. De plus, hormis deux villages uzbeks de cette vallée appartenant au groupe 4, les 5 autres villages uzbeks se positionnent clairement dans le groupe 2. Tandis que les trois villages tadjiks ont tendance à avoir des caractéristiques du groupe 1 (absence de aylâq pour Tadjika) ou du groupe 2 (rareté des surfaces irriguées pour Dch-E Nawi). Or, ces deux groupes rassemblent les villages de la vallée de Jurm, non uzbeks. A l'opposé de la vallée de Khâsh, celle de Jurm se polarise en deux groupes : celui situé en fond de vallée et bénéficiant d'importantes surfaces irriguées (groupe 1), et celui du piémont associant montagne et culture pluviale (groupe 3 ).

\section{Groupe ethnique et espace-ressource}

On vient de montrer la cohérence entre l'origine ethnique et l'espace-ressource des différents villages: les Tadjiks, traditionnellement cultivateurs, habitent les zones basses plutôt irrigables, les Uzbeks, peuple de pasteurs occupent les zones d'altitude adaptées à la pratique de la transhumance et du semi-nomadisme, et les ethnies du bas de l'échelle des valeurs partagées en Afghanistan se situent sur les zones les plus défavorables. Cette situation se retrouve de façon similaire en Iran (15).

Au-delà de cette cohérence, les avatars historiques ont entraîné l'établissement de paysans hors de leur zone agro-écologique de prédilection : c'est le cas des trois villages Chingah, Uzbeks installés en plaine, et du village Tadjika, Tadjiks localisés en montagne. Ces villages ont dû adapter leurs pratiques agricoles au milieu dans lequel ils évoluent, mais cette adaptation n'a pas été poussée jusqu'au bout, et ils ont gardé des caractéristiques fortement liées à leur origine ethnique: Tadjika ne possède aucun aylâq malgré leur proximité du village, alors que les Chingah sont allés chercher loin des habitations des zones d'estives et des parcours hivernaux, permettant les pratiques de semi-nomadisme.

\section{Diversité au sein des villages}

\section{Objectif et méthode}

Au-delà des différences entre villages, il existe traditionnellement en Afghanistan une forte différenciation sociale opposant les zamindar (grands propriétaires) aux petits paysans et aux paysans sans terre. Dans le cadre d'un projet de soutien à l'élevage, il était important d'avoir un aperçu de cette variabilité intra-village afin d'être capable de cerner les véritables bénéficiaires des actions. Cette différenciation sociale se traduit avant tout au niveau des surfaces cultivées. La connaissance de celles-ci pour chaque famille présente de grandes difficultés méthodologiques : les surfaces sont mesurées par la quantité de semence utilisée, et celle-ci varie énormément en fonction du type de terre (irrigable ou non). En outre, étant donné les objectifs poursuivis, l'appréciation des différences inter-familiales s'est faite au travers du cheptel possédé. Une enquête a été menée auprès de 660 familles de 7 villages appartenant aux trois premiers groupes. Pour chaque famille a été noté le nombre d'animaux de chaque espèce et dans le cas des bovins, de chaque type (bœufs, vaches adultes, jeunes de plus d'un an et veaux). Dans un village, l'enquête s'est déroulée dans la totalité des familles (63) et a porté aussi sur la quantité de terre disponible : c'est un village de fond de vallée possédant uniquement des surfaces irriguées, ce qui a facilité l'enquête. Malgré tout, l'ensemble des résultats obtenus doit être considéré avec précaution, car les biais possibles dans les relevés de données quantitatives sont nombreux. Seules, les tendances les plus fortes ont donc été retenues. 


\section{Résultats et discussion}

La taille des troupeaux par famille couvre les valeurs suivantes : bœufs : 0 à 6 ; vaches adultes : 0 à 8 ; petits ruminants : 0 à 300 ; ânes : 0 à 9 . Ces intervalles couvrent l'ensemble des valeurs rencontrées, mais pour 9 familles sur 10 , l'intervalle est beaucoup plus réduit, et s'étend de 0 à 3 pour les boeufs, les vaches adultes et les ânes, et 0 à 15 pour les petits ruminants. De plus, il existe un effet de taille qui se traduit par une augmentation de tous les types d'animaux simultanément : les familles pauvres possèdent peu d'animaux dans chaque espèce, les familles riches possèdent tout à la fois beaucoup de bovins, d'ânes et de petits ruminants (pour les villages pratiquant ce type d'élevage). Ainsi, dans les villages de montagne, les familles qui n'ont pas de boeufs possèdent en moyenne moins de 10 petits ruminants, tandis que celles qui ont 3 boeufs ou plus élèvent plus de 50 petits ruminants.

Enfin, la taille du troupeau et la surface cultivée sont corrélées dans le cas du village où une enquête complète a été effectuée. Ainsi 92 p. 100 des familles sans terre ne possèdent pas de boeufs, et ce chiffre chute à 29 p. 100 pour celles qui possèdent plus de 4 ha. Pour les vaches adultes, cette même tendance existe mais de façon moins marquée. On retrouve une situation largement décrite pour d'autres régions de l'Afghanistan, et qui a été résumée ainsi: "Le cultivateur afghan vit sous le signe du partage inégal" $(5,7)$.

\section{PRATIQUES D'ÉLEVAGE}

L'Afghanistan est un pays à hiver froid et connaît, comme toutes les régions similaires, une période de restriction fourragère, lorsque le sommeil de la végétation et la couverture neigeuse empêchent le recours au pâturage. De plus, l'existence d'un été chaud et très sec oblige les paysans à recourir à des pratiques d'alimentation diversifiées. Dans un tel contexte, la gestion des ressources fourragères semble être une clé d'entrée pertinente pour l'étude des pratiques d'élevage. Les pratiques liées à la reproduction des animaux constituent le deuxième volet important concernant la conduite des élevages. Elles seront présentées en tenant compte de linfluence de la gestion de l'alimentation. Les différents aspects de ces pratiques d'élevage seront détaillés pour chacun des trois premiers groupes de villages tels qu'ils ont été définis précédemment.

\section{Méthodes}

\section{Alimentation}

Les calendriers fourragers : ils ont été définis dans chacun des trois groupes de villages, mais reflètent plutôt une moyenne ; en effet, il a déjà été précisé qu'il existait de fortes disparités au sein d'un même village. Les variations observées seront décrites en complément.

La distribution de fourrage en hiver : celle-ci a été appréciée dans un premier temps sur un aspect qualitatif : liste de tous les aliments utilisés et condition de leur utilisation. Une approche quantitative a été tentée pour la distribution de paille et de trèfle aux bovins. Afin de pouvoir réaliser une appréciation des stocks et des quantités distribuées entre les différentes familles ; le troupeau de chaque famille a été comptabilisé en termes d'unités animales (UA) selon la méthode suivante : boeuf $=1 \mathrm{UA}$, vache adulte et jeune de 3 et 4 ans $=0,6 \mathrm{UA}$, jeune de 2 ans $=0,4$ UA et jeune de 1 an $=0,2$ UA. La connaissance des quantités distribuées et de la valeur alimentaire des rations est très difficile étant donné la variabilité des pratiques et la diversité des ressources utilisées, et n'a pu être réalisée pour les petits ruminants.

L'évolution des états d'engraissement : face à la difficulté de l'approche individuelle du'rationnement et aux limites de l'étude des stocks disponibles dans le cas du recours fréquent au pâturage, le travail sur l'alimentation a été complété par une notation de l'état corporel des animaux. Cette méthode repose sur le principe des maniements et consiste à attribuer une note de 0 à 5 à chaque animal en fonction de critères objectifs : l'appréciation de l'état corporel se fait au niveau du ligament sacrotubéral et des deux dernières' côtes pour les bovins (1), de la région lombaire pour les ovins (11) et de la région sternale pour les caprins $(10)$. Le mouton Turki possède une croupe grasse qui lui sert de réserve pour les périodes difficiles. Il a donc été nécessaire de mettre au point une grille de notation en fonction de la taille de la croupe (9). Les notations d'engraissement ont été réalisées lors des visites mensuelles effectuées dans le cadre des suivis. De plus, au cours de la campagne de vaccination, 3500 moutons Turki ont été notés, soit 21 p. 100 de l'effectif de la zone.

\section{Reproduction}

II n'a pas été possible d'obtenir des données quantitatives concernant la reproduction (fécondité, fertilité,...). Seules les pratiques ont été étudiées et seront détaillées.

\section{Villages de fond de vallée}

Ces villages pratiquent une agriculture irriguée sédentaire. L'élevage bovin y prédomine, étroitement associé à l'agriculture. La moyenne du nombre de bovins parr famille est de l'ordre de 4 à 5 . L'élevage des petits ruminants est inexistant.

\section{Alimentation}

Calendrier fourrager: la figure 1 présente le calendrier fourrager. Pour ces villages, les marzars jouent un rôle particulièrement important pour l'alimentation des animaux : ceux-ci y pâturent de juillet à novembre. II semble que plus la surface du marzar collectif d'un village est 

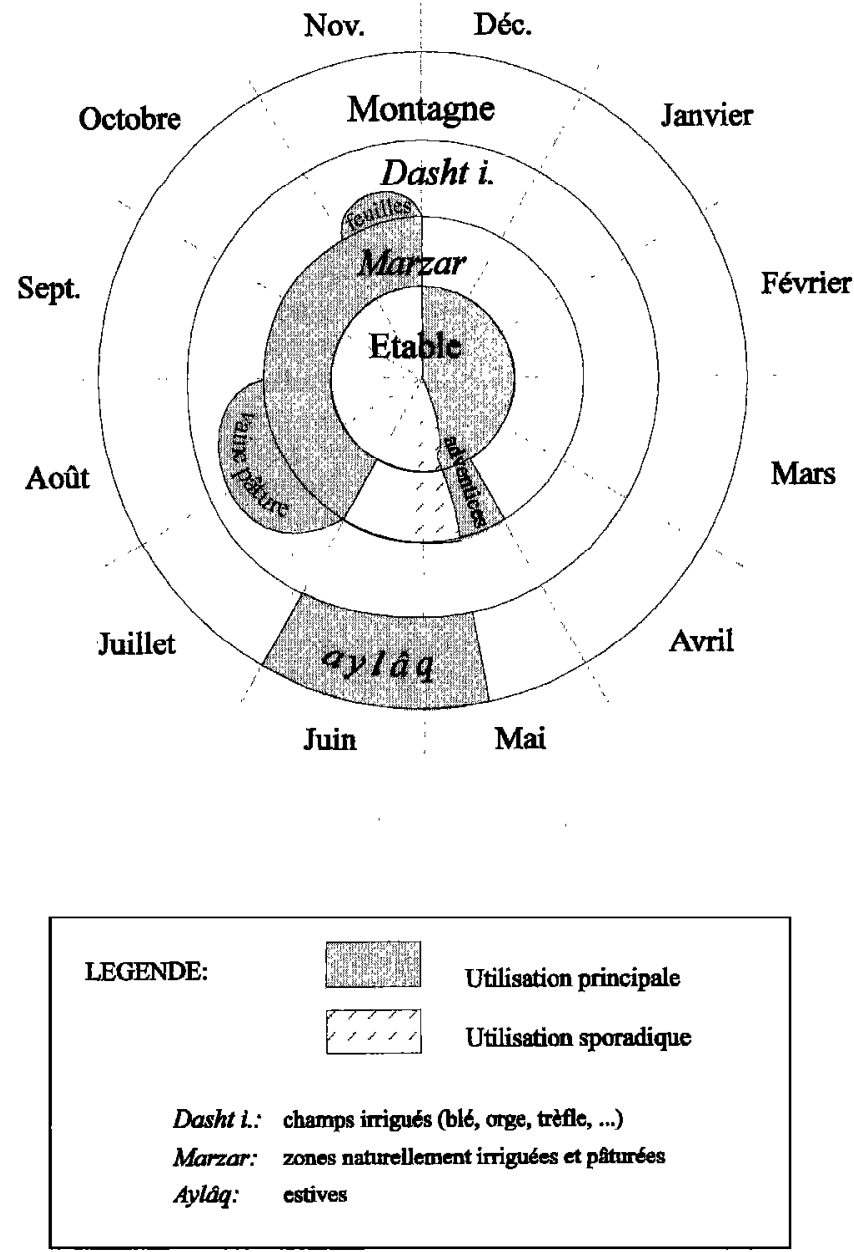

Figure 1: Calendrier d'affouragement des bovins dans les villages de fond de vallée (type 1) et ceux de montagne irrigable (type 2).

grande, plus la taille des troupeaux est importante. Au mois de novembre, lorsque les ressources du marzar s'épuisent, les feuilles des arbres sont collectées systématiquement et distribuées aux animaux. De décembre à avril, les bovins sont en stabulation complète et sont nourris de paille et de trèfle. Ces deux fourrages sont hachés finement et mélangés avant d'être distribués. Au début du printemps, alors que les stocks s'épuisent, les enfants pratiquent une activité intense de désherbage des champs de blé et ramassent soigneusement les adventices qui sont distribuées aux bovins. L'association agriculture/élevage apparaît là sous un angle inhabituel. Pendant une courte période (mi-mai à fin juin), ces villages de fond de vallée ont recours aux aylâq sur lesquels les vaches allaitantes et les jeunes bovins vont pâturer. Les boeufs restent au village car c'est une période de travail intense. Après la récolte de trèfle (fin juillet) et avant les labours d'automne (fin septembre), les animaux sont laissés en vaine pâture sur les champs, et sont regroupés pour l'occasion en petits troupeaux col- lectifs. Après cette période et jusqu'à la récolte de trèfle, le pâturage est strictement interdit en dehors des marzars.

Il existe donc des périodes de pâturage collectif (marzar, aylâq, vaine pâture), et des périodes consacrées à la récolte de ressources' "publiques" (adventices, feuilles). Les familles les plus pauvres, c'est-à-dire celles qui n'ont pas de terre, exploitent au maximum ces ressources collectives. Les plus riches essaient au contraire de garder les animaux le plus possible en stabulation afin de veiller à leur alimentation.

Distribution de fourrages en hiver : la paille de blé et le trèfle persan constituent la base de l'alimentation des bovins. La paille des vesces cultivées est utilisée lorsqu'elle est disponible. Les familles qui possèdent des marzars suffisamment grands peuvent distribuer un peu de foin. Les vaches ne reçoivent de complément que pendant le début de la lactation, rarement avant. En revanche, les boeufs font l'objet de soins très attentifs: ils reçoivent systématiquement un complément alimentaire à base de tourteau (pavot, lin ou moutarde) ou de farine de légumineuses (vesce ou pois), parfois associé à du son. Les boeufs ne sont jamais envoyés en pâturage. En début de saison des labours, les paysans distribuent parfois de l'orge pour préparer les animaux. L'alimentation du jeune veau est soumise à la concurrence avec l'homme ; les éleveurs le sèvrent souvent le plus rapidement possible, ce qui provoque, dans les 6 premiers mois, un certain marasme aggravé par le parasitisme.

Les stocks de paille disponibles pour l'hiver varient de 700 à $1600 \mathrm{~kg}$ par UA avec une moyenne de 1000 $\mathrm{kg} / \mathrm{UA}$ par famille. Si l'on considère qu'il y a environ 110 jours de stabulation, cela représente $9,5 \mathrm{~kg}$ de paille par jour pour un boeuf de $300 \mathrm{~kg}$ auquel il faut ajouter 1,5 à 2 $\mathrm{kg}$ de trèfle. Cette ration apparaît correcte sur le plan énergétique, déficitaire en azote et excédentaire en calcium. L'apport de tourteau ou de farine $(0,5$ à $1 \mathrm{~kg} / \mathrm{jour})$ permet une certaine correction de cette ration. La distribution de son de blé rééquilibre, dans une certaine mesure, l'alimentation minérale. Cette distribution de complément explique peut-être que les paysans ne signalent pas de problèmes importants de la reproduction, contrairement à d'autres régions d'Afghanistan.

Evolution des états corporels : les notations ont été réalisées sur un faible nombre d'animaux (19 boeufs et 20 vaches) et les résultats ne sont pas, d'emblée, généralisables. Néanmoins, ils fournissent des données qui confirment les analyses faites sur la distribution hivernale de fourrage:

- meilleur état des boeufs (moyenne 2,8 en novembre) que des vaches $(2,07$ à la même période), qui est à mettre en relation avec une distribution permanente de complément aux animaux de trait ;

- meilleur état d'engraissement des vaches dans les troupeaux qui comportent des boeufs $(2,34)$ que dans les 
troupeaux sans boeufs $(1,58)$. Or, en moyenne, les familles propriétaires de boeufs possèdent de la terre et donc des ressources fourragères (paille, trèfle,...), alors que les paysans sans terre doivent acheter l'ensemble du fourrage des bovins ;

- il n'y a pas d'évolution de l'état d'engraissement des animaux au cours de l'hiver. Les ressources fourragères semblent globalement suffisantes, et les problèmes d'amaigrissement ou de carences sont plutôt liés au stade physiologique qu'à la saison de l'année : ce sont les vaches en début de lactation dont l'état général s'altère, et cette situation est à mettre en relation avec l'absence de préparation au vêlage.

\section{Reproduction}

Les boeufs sont castrés à l'âge de 4-5 ans. Cet âge tardif entraîne la présence d'un grand nombre de jeunes mâles aptes à la reproduction. L'existence de pratiques collectives de mai à novembre favorise un mélange des animaux, et permet aux familles possédant seulement une ou deux vaches de disposer de reproducteurs, tout en évitant des problèmes de consanguinité. En revanche, ce système empêche toute pratique de choix du reproducteur et de sélection. La période de saillie se situe surtout en août et septembre, et les mises bas interviennent essentiellement au printemps.

\section{Villages de montagne irriguée}

Ce sont des villages qui associent agriculture sédentaire et élevage transhumant. L'élevage bovin est conduit de la même façon que dans les villages de fond de vallée. Les effectifs sont similaires et l'alternance marzar/stabulation hivernale est semblable. Néanmoins, ces villages se caractérisent par un élevage de petits ruminants important : la moyenne des troupeaux par famille est de 25 têtes. Seules les données concernant les petits ruminants seront donc présentées dans ce chapitre.

\section{Alimentation}

Calendrier fourrager (fig. 2) : l'utilisation maximale des ressources fourragères offertes par la montagne est la caractéristique de l'élevage de petits ruminants. Ils passent 7 mois sur 12 dans les aylâq. Les 5 autres mois, ils pâturent la montagne proche tant que la neige ne recouvre pas totalement les pentes. La durée de stabulation complète est variable selon l'altitude des villages et, bien sûr, la longueur de l'hiver. En 1990, elle s'est étendue sur 1 à 2 mois. Même durant cette période, les petits ruminants utilisent les ressources de la montagne puisqu'ils consomment exclusivement du yekhan récolté l'été

1. II n'a pas été possible d'effectuer la détermination botanique de cette plante.
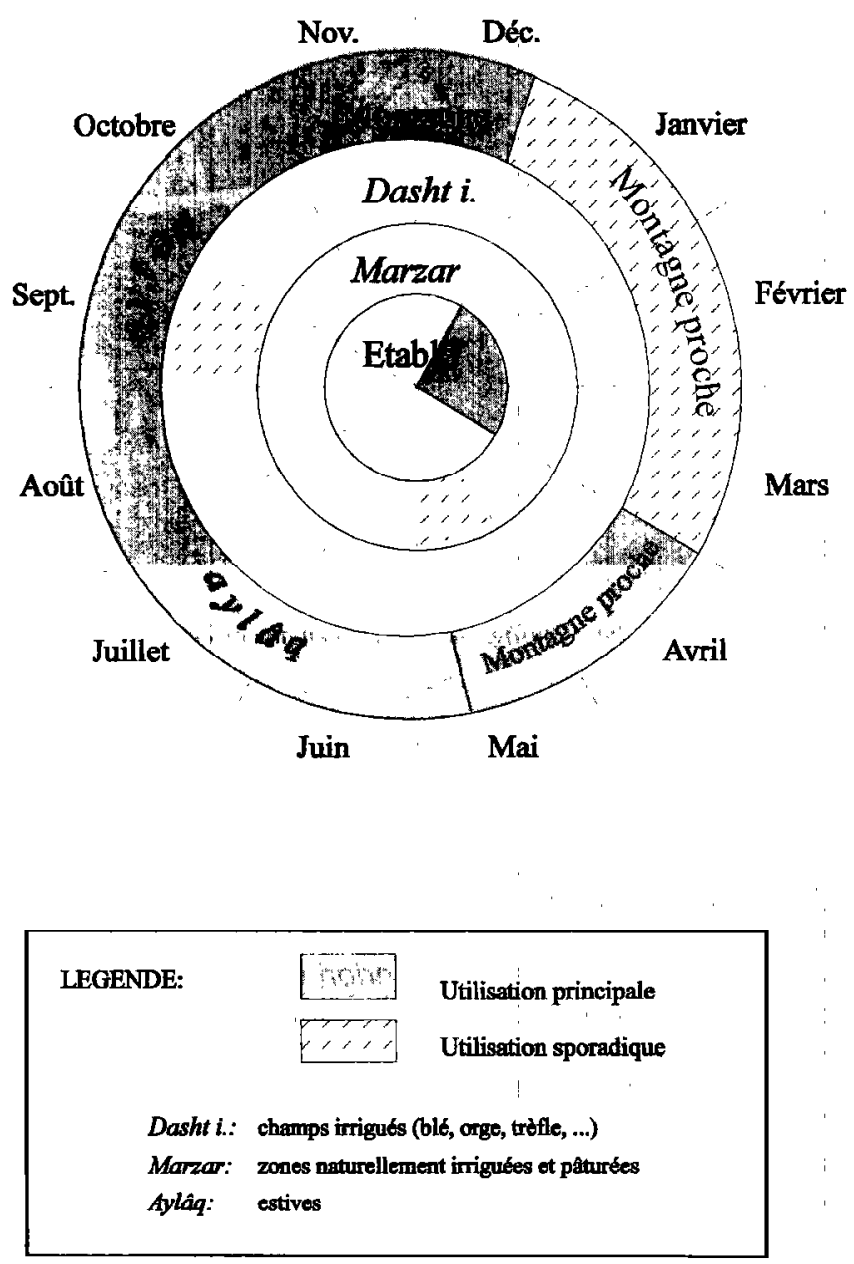

Figure 2 : Calendrier d'affouragement des petits ruminants dans les villages de montagne irrigable (type 2) et ceux de montagne sèche (type 3).

en montagne. Dans de rares occasions, les petits ruminants recourent au marzar et à la vaine pâture sur le dasht. II faut noter que les caprins, considérés comme plus sensibles au froid, sont redescendus un mois plus tôt que les moutons des aylâq. Quelques troupeaux restent tout au long de l'hiver dans les zones intermédiaires entre les villages et les estives appelées qâtan. Ce sont en général des troupeaux de taille importante appartenant à des gros propriétaires. Ces troupeaux, élevés uniquement à une fin bouchère, recourent au pâturage durant tout l'hiver. La distribution de fourrage à ces troupeaux est limitée au maximum.

La distribution de fourrage en hiver : le fourrage de montagne est l'aliment essentiel des petits ruminants au cours des périodes de stabulation. Il est haché à la main, et distribué de 5 à 8 fois par jour lorsque les animaux ne sortent pas du tout. Si le pâturage est possible, les petits ruminants reçoivent du fourrage une ou deux fois par jour. Les éleveurs qui en disposent distribuent la paille des légumineuses. La paille de blé ou d'orge n'est jamais distribuée aux petits ruminants. 


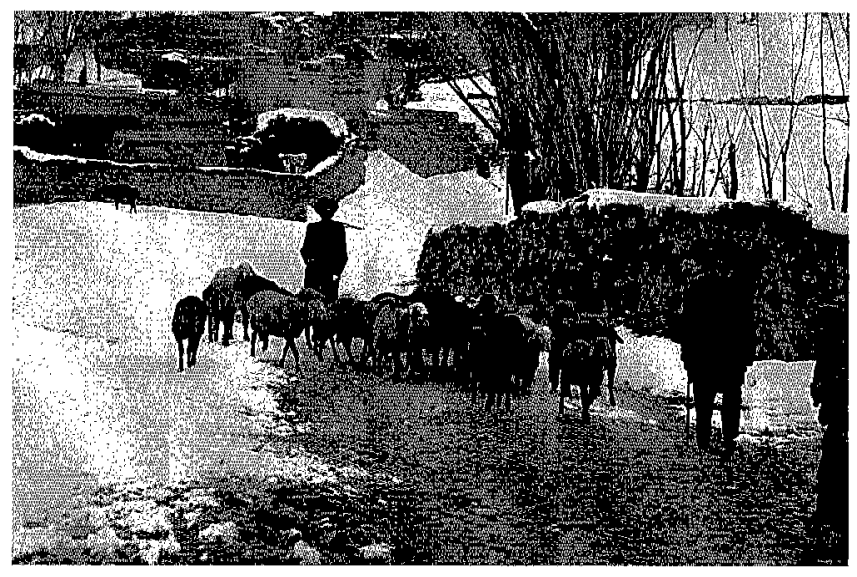

Photo 2 : Afghanistan. Pâturage des moutons en hiver (février 1990).

L'allotement, pratique fréquente notamment dans les grands troupeaux, se fait soit a priori (séparation des brebis en gestation), soit a posteriori (séparation des animaux maigres). II intervient à l'automne (descente plus précoce des animaux en mauvais état) ou au printemps. Dans ce dernier cas, il concerne surtout les mères en fin de gestation et début de lactation. Elles restent en bergerie et reçoivent du foin de trèfle, tandis que le troupeau part pâturer. Les jeunes sont rapidement sevrés et, à cette fin, disposent dès les premiers jours de fourrage en libre service. Dès l'âge de 1 mois, les enfants les emmènent pâturer à proximité du village.

\section{Evolution des états corporels}

- Ovins : au sein de chaque village, selon les troupeaux, les valeurs sont très variables, néanmoins les moyennes par village montrent une décroissance linéaire de la note d'état corporel attribuée à la croupe : celle-ci passe de 2,57 (moyenne des troupeaux notés dans un village) au début du mois de janvier à 1,42 à la fin du mois de mars. Pour les troupeaux en suivi, notés sur un laps de temps plus large, les valeurs s'étendent de 2,8 au 20 décembre à 1,19 au 30 avril. Cette décroissance débute dès le retour des troupeaux au village à la descente des aylâa au mois de décembre ; cela semble montrer que le rôle de la croupe grasse comme réserve hivernale est intégré dans la logique d'affouragement. Durant le mois de février, qui a été le seul mois de stabulation complète, un répit dans cette décroissance a été observé.

Les troupeaux élevés dans les qâtan durant l'hiver présentent une moyenne systématiquement plus élevée d'un demi-point par rapport à ceux en stabulation dans les villages.

- Caprins : seuls les troupeaux en suivi ont été notés. Ils représentent donc un effectif faible. La note attribuée montre une décroissance similaire à celle des ovins, suivi d'un répit durant la période de stabulation. La notation, poursuivie jusqu'au mois de mai, semble indiquer que c'est au mois d'avril que l'état corporel des animaux est au plus bas.

\section{Reproduction}

La période de lutte se situe en octobre et novembre alors que les animaux pâturent en troupeaux villageois dans les aylâq. Les béliers sont toujours de race Turki. Cette pratique permet aux petits éleveurs de disposer de reproducteurs de bonne qualité, que seuls de plus gros éleveurs peuvent sélectionner au sein d'un grand troupeau. Un paysan peu fortuné mais désirant se constituer un troupeau de moutons, achète des animaux de race Gadi moins coûteux que les Turki. Au fil des années, grâce à la reproduction dans le troupeau collectif, ces éleveurs aboutissent progressivement à une "turkisation" de leurs animaux. Ce mécanisme permet d'éviter un investissement trop important, tout en aboutissant à un troupeau Turki.

Les mises bas se font aux mois de mars et avril, et semblent très groupées. Or, à cette période de l'année, l'état corporel des brebis est au plus bas et les réserves fourragères sont épuisées; la sous-alimentation des agneaux et des chevreaux est la première cause de mortalité néonatale. Des flambées d'avortement sont signalées dans certains troupeaux en rapport avec des épizooties de pasteurellose. Hormis ce problème, aucun trouble de la reproduction n'est évoqué par les paysans comme un problème majeur.

\section{Les villages de montagne sèche}

Ils associent la culture pluviale du blé et de l'orge à l'élevage des petits ruminants. Les champs sont situés autour du village ou dans la montagne. Certains de ces villages pratiquent une activité de semi-nomadisme, qui concerne tout ou partie du village. De juin à septembre, les paysans vont habiter dans les aylâq, et y pratiquent tout à la fois culture et élevage. L'élevage des petits ruminants présente les mêmes caractéristiques que celui des villages de montagne irriguée, à quelques différences près qui seront évoquées ci-dessous.

\section{Alimentation}

L'élevage bovin est largement conditionné par la rareté ou l'absence de surfaces irrigables naturellement ou artificiellement. La moyenne des troupeaux par famille est d'environ deux bovins.

Calendrier fourrager (figure 3 ) : le recours au pâturage sur marzar est impossible ou très limité. Le calendrier fourrager se partage donc de façon à peu près égale entre la stabulation de décembre à avril, la montagne d'avril à juin 


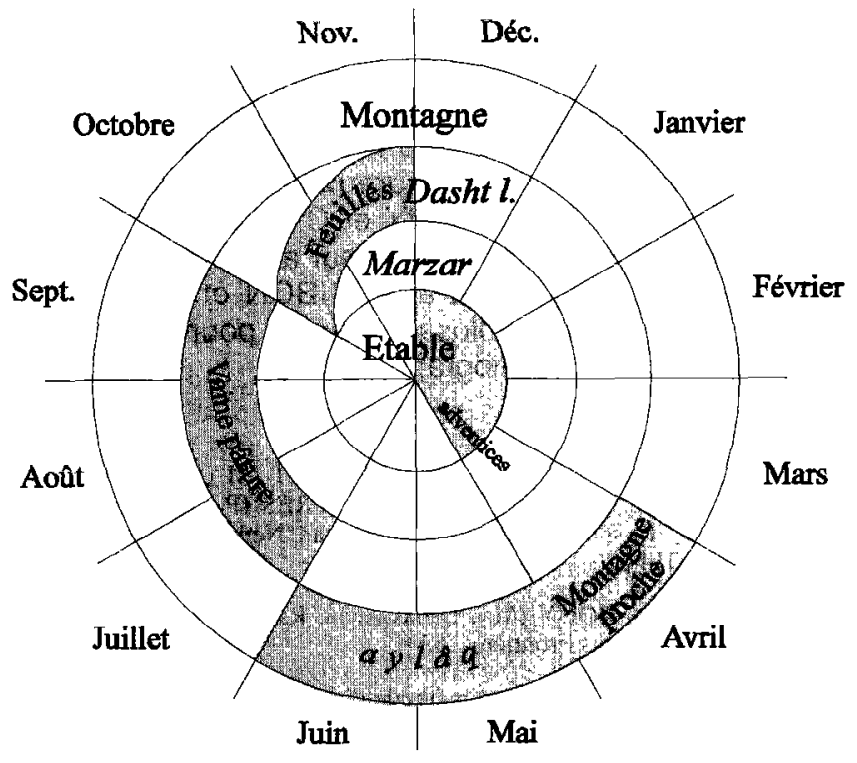

\begin{tabular}{|lll|}
\hline LEGENDE: & Utilisation principale \\
& & \\
Dasht $l .:$ & champs en culture pluviale (ble, orge) \\
Marzar: & zones naturollement irriguées et paturées \\
Aylaq: & estives \\
\hline
\end{tabular}

Figure 3 : Calendrier d'affouragement des bovins dans les villages de montagne sèche (type 3).

et la vaine pâture de juillet à octobre. Cette vaine pâture est remplacée par du pâturage dans les aylâq pour les familles qui sont en montagne à cette période.

Distribution de fourrage : les paysans de ces villages disposent de très peu de fourrage (trèfle, luzerne, toin de marzar, paille de légumineuses) du fait de la prédominance du domaine pluvial. La paille de blé demeure le seul aliment des bovins durant l'hiver. A la fin de la mauvaise saison, si les stocks de paille sont épuisés, certains paysans distribuent du yekhan, qui est un fourrage réservé aux petits ruminants. Quand on connaît la rigueur des éleveurs afghans dans l'attribution d'un fourrage à chaque espèce (paille et trèfle aux bovins, yekhan aux petits ruminants, luzerne aux ânes, orge aux chevaux), la distribution d'un fourrage de montagne aux bovins signale une période de soudure difficile dans ces villages.

Etat corporel : aucun animal n'a été noté dans ces villages.

\section{Reproduction}

Les pratiques concernant l'élevage des petits ruminants sont très similaires à celles observées dans les villages de montagne irriguée. Mais l'absence ou la rareté des surfaces irriguées interdit de complémenter, au printemps, les animaux qui en auraient besoin, notamment les femelles en début de lactation. De fait, c'est dans ce type de village qu'ont été observés les cas les plus importants de mortalité néonatale des agneaux en relation avec un mauvais état corporel des mères. Cette contrainte fourragère a favorisé l'exploitation par ces villages de la montagne proche par le biais des qâtan dans lesquels la totalité des troupeaux du village sont gardés par un berger salarié jusqu'à la fin du mois de décembre. Ces qâtan collectifs permettent à un plus grand nombre de paysans de maintenir leurs animaux en montagne plus longtemps que ceux des villages de montagne irriguée. Les pratiques touchant à la reproduction des petits ruminants sont identiques à celles des villages du groupe 2 .

\section{CONCLUSION}

La région de Jurm en Afghanistan offre, dans un espace géographique relativement réduit, une grande diversité de situations, tant naturelles qu'humaines. Les contraintes d'altitude et d'irrigation, combinées aux origines ethniques et à l'histoire de chaque village, ont favorisé la coexistence de villages d'agriculteurs sédentaires et de villages d'agropasteurs semi-nomades. Néanmoins, la culture du blé reste l'élément fondamental, commun à ces populations.

L'élevage bovin est fortement associé au cycle des grandes cultures, blé, orge et trèfle. Les boeufs fournissent le travail, et les champs irrigués apportent la quasitotalité des fourrages destinés aux bovins. Cet élevage est donc relativement développé dans les villages disposant de surfaces irriguées importantes. Plus réduit dans les autres villages, l'élevage bovin est limité au strict minimum nécessité par le labour et permis par les ressources en paille. Dans la majorité des cas, la taille du troupeau de bovins d'une famille est adaptée aux ressources fourragères dont elle dispose. Les cas de sousnutrition sont à mettre en rapport avec l'absence d'adaptation de la ration à l'état physiologique des femelles (gestation, lactation) et la concurrence entre l'homme et le veau pour la consommation du lait. A contrario, le bœeuf, animal emblématique du paysan propriétaire, bénéficie d'une alimentation adaptée à la variabilité de ses besoins : entretien, travail léger, travail intense.'

L'élevage des petits ruminants est complètement déconnecté des zones cultivées, et l'ensemble des ressources fourragères provient de la montagne. Ces troupeaux réalisent quand même un transfert de fertilité des estives et de la montagne proche vers les champs, grâce au fumier accumulé durant l'hiver en stabulation. En effet, seul ce fumier et les excréments humains sont utilisés comme fertilisant. Les bouses des bovins sont mises à sécher 
sous forme de galettes, utilisées comme combustible. Les pratiques d'alimentation des petits ruminants s'appuient largement sur les capacités des animaux à stocker des réserves dans leur croupe grasse, et à les utiliser tout au long de l'hiver. Mais les mises bas interviennent au printemps, alors que les animaux sont au plus faible niveau d'engraissement, ce qui a, pour certains élevages, des conséquences dramatiques. La possibilité pour un éleveur de faire passer l'hiver et le printemps à son troupeau dans de bonnes conditions dépend de nombreux facteurs : durée de l'hiver, enneigement hivernal, réserves graisseuses accumulées et stocks de fourrages constitués durant l'été et l'automne. Ces deux derniers éléments sont liés à l'existence de pratiques collectives, puisque les estives pâturées ou fauchées sont communautaires. Cette gestion collective différencie l'élevage des petits ruminants de celui des bovins qui relève beaucoup plus d'une gestion individuelle.

\section{BIBLIOGRAPHIE}

1. AGABRIEL (J.), GIRAUD (J.M.), PETIT (M.). Détermination et utilisation de la note d'état d'engraissement en élevage allaitant. Bull. tech. Cent. Rech. Zoot. vét. Theix, 1986 (66) : 43-50.

2. BAI I.AND (D.). Bârân. In : YARSHATER (E.). ed. Encyclopedia Iranica, vol. III, fasc. 7, Banân-Bardesanes. London, Routledge et Kegan Paul. 1989.

3. BALLAND (D.). Fromages traditionnels et fromages industriels d'Afghanistan. Prod. past. Soc., 1985, 17 : 13-26.

4. BOURBOUZE (A.), DONADIEU (R.). L'élevage sur parcours en régions méditerranéennes. Montpellier, CIHEAM/IAM, 1987. 104 p.

5. CENTLIVRES (P.), CENTLIVRES-DEMONT (M.). La société afghane, structure et valeurs. In : Afghanistan, la colonisation impossible. Paris, Ed. du Cerf, 1984. p. 57-80.

6. CENTLIVRES (P.). Identité et image de l'autre dans l'anthropologie populaire. In : CENTLIVRES (P.), CENTLIVRES-DEMONT (M.). Et $\mathrm{s}$ on parlait de l'Afghanistan. Neuchâtel, Institut d'ethnologie, 1988. $314 \mathrm{p}$.

7. CENTLIVRES (P.), CENTLIVRES-DEMONT (M.). La société afghane forme d'organisation et valeurs. In : CENTLIVRES (P.), CENT LIVRES-DEMONT (M.). El si un parlaiit de l'Afghanistan. Neuchâtel, Institut d'ethnologie, 1988. $314 \mathrm{p}$

BOUY (M.), DASNIĖRE (J.). Typology of villages and livestock rea ring practices in Badakhshan (north-east Afghanistan). Revue Elev. Méd. vét. Pays trop., 1994, 47 (2) : 245-256

The livestock rearing system was studied in two valleys in Badakhshan, north-east Afghanistan. The first part of the study involved establishing a typology of the villages, taking into account their agroecological and human aspects. This typology revealed three main groups: irrigated valley bottom, irrigated mountain and dry mountain. On these grounds, the authors present a study of the livestock rearing practices in terms of the feeding and reproduction of bovines and small ruminants. These practices show a strong link between cattle rearing and arable cultivation, and exclusive exploitation of the mountainous areas by small ruminants. The role of collective practices is studied.

Key words : Ass - Cattle - Goat - Horse - Hen - Sheep - Highlands Valley - Village - Mixed farming - Animal husbandry method - Reproduction - Feeding - Fodder - Fattening - Nomadism - Pastoralism - Livestock economics - Afghanistan.

\section{REMERCIEMENTS}

Nous adressons nos remerciements à D. BALLAND (CNRS, Sciences Sociales du monde iranien contemporain), pour son accueil chaleureux et la documentation sur l'Afghanistan, à M. JARRY et C. WAGNER (IBEAS, Université de Pau, France) pour leur aide précieuse dans l'analyse des données et à A. GIBON et G. BALENT (INRA-SAD, Castanet-Tolosan, France) pour leur apports méthodologiques et bibliographiques.

8. CENTLIVRES (P.), CENTLIVRES-DEMONT (M.). Les fondeurs de socs à araire et de marmites. In : CENTLIVRES (P.), CENTLIVRESDEMONT (M.). Et si on parlait de l'Afghanistan. Neuchâtel, Institut d'ethnologie, $1988.314 \mathrm{p}$.

9. DASNIERE (J.), BOUY (M.). Badakhshan, Rapport de mission, 1990. Lyon, Vétérinaires Sans Frontières. 53 p.

10. Essai de mise au point d'une méthode d'estimation de l'état d'engraissement des chèvres corses. Séminaire FAO, Sous-Réseau Caprin : Groupe Nutrition de la Chèvre Laitière, Grangeneuve, Suisse, 16-18 octobre 1984.

11. GIBON (A.), DEDIEU (B.), THERIEZ (M.). Les réserves corporelles des brebis. Stockage, mobilisation et rôle dans les élevages de milieu difficile. In: Xe journées de la Recherche ovine et caprine, Paris, ITOVIC, 1985. p. 178-211.

12. MAWLAWI BORHAN-AL-DIN KHAN KOSHKAKI, Râhnamâye Qataghan wa Badakh-shan 1302/1923. Traduction française: REUT (M.). Paris, Qataghan et Badakhshan, 3 vol., 1979.

13. OSMAN (A.H.). Proche-Orient : amélioration des races ovines. Revue mond. Zootech., 1985, $54: 2-15$.

14. PIALOT (D.), CHESSEL (D.), AUDA (Y.). Description de milieu et analyse factorielle de correspondances multiples. c.r. Acad. Sci., Paris, 1984, 298, série III. p. 309-314.

15. PLANHOL (X. DE). Les fondements géographiques de l'histoire de l'Islam, Paris, Flammarion, 1968, $442 \mathrm{p}$

16. PLANHOL (X. DE), Badakhshân. In :YARSHATER (E.). ed. Encyclopedia Iranica. vol. III, fasc. 4, Bacha-e saqaw-Bahai faith III. London, Routledge et Kegan Paul, 1989.

17. POULTON (M.), POULTON (R.), Ri-jang : un village tajik dans le nord de l'Afghanistan, Thèse EHESS, Paris, 1979.

BOUY (M.), DASNIÈRE' (J.). Tipología de los pueblos y präcticas de crianza en la zona de Badakhshan (nordeste de Afganistán). Revue Élev. Méd. vét. Pays trop., 1994, 47 (2) : 245-256

Un estudio sobre los sistemas de crianza fue llevado a cabo en dos valles de la zona de Badakhshan; región nordeste de Afganistán. La primer etapa del estudio consistió en establecer una tipología de los pueblos, considerando tanto aspectos agro-ecológicos, como humanos. Esta tipología permitió determinar tres grupos principales: valle irrigado, montaña irrigada y montaña seca. En base a esta tipología, se presentó un estudio sobre las prácticas de manejo, considerando la alimentación y la reproducción de los bovinos y pequeños rumiantes de la zona. Las prácticas muestran una fuerte asociación, por un lado entre la crianza bovina y la agricultura y por otro de la explotación exclusiva de los pequeños rumiantes en zonas montañosas. En cada caso se discute el papel de las prácticas colectivas.

Palabras clave : Asno - Bovino - Caprino - Caballo - Gallina - Ovino Zona de montaña - Valle - Pueblo - Asociación agricultura - Ganaderia Metodo de crianza - Reproducción - Alimentación - Forraje - Engorde Nomadismo - Pastoralismo - Economía de la cría - Afganistán. 